\title{
Effect of $r$-Irradiation on the Crystalline Transition Temperature in Poly(tetrafluoroethylene)
}

\author{
Mituaki Tutrya \\ Tokyo Metropolitan Isotope Research Center, \\ 2-11-1, Fukazawa, Setagaya, Tokyo.
}

(Received June 12, 1973)

\begin{abstract}
The effect of $\gamma$-irradiation on the $19^{\circ} \mathrm{C}$ transition of poly(tetrafluoroethylene) was studied with broad-line NMR and the transition temperature was measured as a function of total dose up to $10^{9} \mathrm{R}$. The transition temperature is lowered with increasing dose in all the following cases: (a) room-temperature irradiation, (b) roomtemperature irradiation with subsequent annealing, and (c) irradiation above the melting point. In case (b), the lowering of the transition temperature is attributed to the mainchain scission in the crystalline phase. In case (a), in addition to the scission, the occlusion of degradation products is effective, and in case (c), the formation of sidebranches $\left(-\mathrm{CF}_{3}\right)$ plays an important role. The results are analysed by a theory of rotational phase transition and a satisfactory agreement between theory and experiment is obtained.
\end{abstract}

KEY WORDS $\gamma$-Irradiation / NMR / Transition / Poly(tetrafluoroethylene) / Degradation Theory / Chain Scission / Degradation Products / Hexafluoropropylene /

Poly(tetrafluoroethylene) (PTFE) has drawn attention as a typical degradation-type polymer for $\gamma$-irradiation. ${ }^{1}$ For radiation doses up to $10^{8} \mathrm{R}$, the radiation-induced changes of molecular motion take place predominantly in the amorphous phase. ${ }^{2-6}$

On the contrary above $10^{8} \mathrm{R}$, the degree of crystallinity decreases ${ }^{7,8}$ and the crystalline transition temperature is remarkably lowered with increasing dose..$^{8-12}$ Licht and Kline ${ }^{8}$ proposed an empirical equation of the decrease in the transition temperature with increasing dose. They have suggested that the decrease in the transition temperature is associated with a decrease in the average molecular weight.

In our previous paper, ${ }^{9,13}$ NMR results have been reported on radiation-induced changes of the transition temperature $T_{\mathrm{t}}$ in PTFE: (a) $T_{\mathrm{t}}$ lowers by about $60^{\circ} \mathrm{K}$ when irradiated with $8.9 \times 10^{8} \mathrm{R}$ at room temperature in air. ${ }^{9}$ (b) When annealed at $325^{\circ} \mathrm{C}$ after irradiation in air, $T_{\mathrm{t}}$ shifts back by about $20^{\circ} \mathrm{K} .{ }^{9}$ (c) When irradiated with $1.1 \times$ $10^{7} \mathrm{R}$ in a vacuum-sealed glass tube, the lowering of $T_{\mathrm{t}}$ is the most noticeable at $340^{\circ} \mathrm{C}$ irradiation. ${ }^{13}$ The mechanisms of these results have been suggested by the present author as the main-chain scission in the crystalline phase, the occlusion of degradation products, and the removal of these products with annealing. ${ }^{9}$

In this paper, further experiments are described on the lowering of $T_{\mathrm{t}}$ as a function of total dose and the mechanisms are discussed more quantitatively on the basis of a theory of the rotational phase transition. A satisfactory agreement between theory and experiment on the effects of chain scission and degradation products is obtained.

\section{EXPERIMENTAL}

PTFE samples and NMR instruments used in this experiment were the same as those in the previous papers. ${ }^{9,13}$

Samples were exposed to ${ }^{60} \mathrm{Co} \gamma$-rays at room temperature in air at dose rates of $8.6 \times 10^{5} \mathrm{R} / \mathrm{hr}$ to $2.1 \times 10^{6} \mathrm{R} / \mathrm{hr}$ up to $1.1 \times 10^{9} \mathrm{R}$. The crystalline transition temperature $T_{\mathrm{t}}$ in PTFE was obtained from the measurement of temperature dependence of NMR spectra. $T_{\mathrm{t}}$ was assumed to be the temperature where abrupt narrowing of the broad component starts. ${ }^{3,14} \quad T_{\mathrm{t}}$ thus defined was $19^{\circ} \mathrm{C}$ or slightly lower in the original sample before irradiation. When irradiated above $10^{9} \mathrm{R}, T_{\mathrm{t}}$ 


\section{TUtiYA}

became difficult to determine experimentally, ${ }^{9}$ because the NMR line shapes could not be decomposed distinctly into broad and narrow components. After the $T_{\mathrm{t}}$ measurement, the samples were annealed at temperatures 300 to $325^{\circ} \mathrm{C}$ in air. $^{9}$

Another series of samples were irradiated above room temperature in vacuum-sealed glass tubes at a dose rate of $5.5 \times 10^{5} \mathrm{R} / \mathrm{hr} .^{13}$

\section{RESULTS}

Figure 1 shows the crystalline transition temperature $T_{\mathrm{t}}$ plotted as a function of total dose for two series of PTFE samples, one irradiated at room temperature in air and the other irradiated and subsequently annealed in the vicinity of its melting point in air. The data reported by Licht and Kline ${ }^{8}$ for PTFE irradiated at room temperature in air are also plotted in Figure 1 for comparison.

In Table I are summarized the results of hightemperature irradiation in vacuum-sealed glass tubes. The fourth column of Table $I$ indicates the weight fraction of hexafluoropropylene (HFP). ${ }^{15}$ The formation of side-branches $\left(-\mathrm{CH}_{3}\right)$ is verified by the observation of an absorption

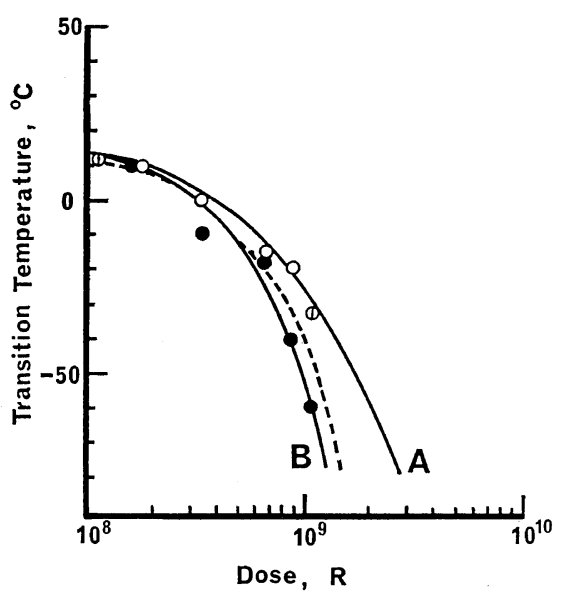

Figure 1. Variation of the crystalline transition temperature with total dose for PTFE samples: $(\odot)$, irradiated at room temperature in air; (O), irradiated in air and subsequently annealed; (1), the data by Licht and Kline. ${ }^{8}$ The solid curves A and B represent the theoretical values of eq 17 and 19, respectively. The dashed line represents Licht-Kline's equation, eq 20.
Table I. Transition temperature and hexafluoropropylene content (wt \%) of PTFE samples irradiated in vacuum-sealed glass tubes

\begin{tabular}{cccl}
\hline $\begin{array}{c}\text { Total dose, } \\
\mathbf{R}\end{array}$ & $\begin{array}{c}\text { Irradiation } \\
\text { temperature, } \\
{ }^{\circ} \mathbf{C}\end{array}$ & $\begin{array}{c}\text { Transition } \\
\text { temperature, }\end{array}$ & $\begin{array}{c}W_{\mathbf{H}} \\
\%\end{array}$ \\
\hline $1.1 \times 10^{7}$ & 150 & 288 & 0 \\
& 300 & 288 & 0 \\
& 330 & 273 & 0.5 \\
& 340 & 273 & 0.4 \\
& 360 & 283 & 0 \\
& 370 & 288 & 0 \\
$6.2 \times 10^{7}$ & 300 & 273 & 2.6 \\
$1.1 \times 10^{8}$ & $310 \sim 340$ & & 3.4 \\
$1.8 \times 10^{8}$ & 25 & 283 & 0 \\
\hline
\end{tabular}

peak at $10.2 \mu$ in the infrared spectrum. ${ }^{15}$

\section{THEORETICAL}

\section{Effect of Main-Chain Scission on the Transition Temperature}

As has been well established, ${ }^{16}$ the $19^{\circ} \mathrm{C}$ transition in PTFE is a rotational phase transition, ${ }^{17}$ above which the molecular chains are aligned in the hexagonal symmetry with a rotational motion around the chain axis. On the other hand below $19^{\circ} \mathrm{C}$, the chains are arranged in the pseudohexagonal lattice.

The transition temperature $T_{\mathrm{t}}$ is in general determined by the increment of enthalpy $\Delta H$ from below to above $T_{\mathrm{t}}$ and that of entropy $\Delta S$ as

$$
T_{\mathrm{t}}=\frac{\Delta H}{\Delta S}
$$

As a simplest model, a chain is assumed to consist of rigid segments, each of which involves $n \mathrm{CF}_{2}$ groups. In the rotational state $\mathrm{B}$ above $T_{\mathrm{t}}$ the segment rotates around the chain axis, but in the rigid state $\mathrm{A}$ below $T_{\mathrm{t}}$ the rotation is frozen. The increment of entropy of a segment at the transition from $\mathrm{A}$ to $\mathrm{B}$ is thus a constant $k$. The enthalpy increase of a segment is given by

$$
\Delta H=n \Delta E
$$

where $\Delta E$ is the energy increment of each $\mathrm{CF}_{2}$ group in the segment. Then $T_{\mathrm{t}}$ in eq 1 is written in the form

$$
T_{\mathrm{t}}=\frac{n \Delta E}{k}
$$


As discussed by Wada, et al., ${ }^{18}$ the length of a segment is determined by the balance between intrachain and interchain forces of $\mathrm{C}-\mathrm{C}$-bond torsion and given approximately by

$$
n=\sqrt{\frac{f}{g}}
$$

where $f$ and $g$ are intrachain and interchain torsional force constants of the $\mathrm{C}-\mathrm{C}$ bond, respectively. The value of $n$ for PTFE has been evaluated by Itani and $\mathrm{Wada}^{19}$ as $n=4$ from the Grüneisen constant. When one end of a segment is free, $n$ is smaller than that given by eq 4 and may be assumed as half of $n$ in eq 4 .

Let us consider a crystal consisting of $m \mathrm{CF}_{2}$ groups or $m / n$ segments. After irradiation with a dose $D$, the main-chain scission occurs in $m r D$ bonds, where $r$ is the probability of main-chain scission per unit dose. The number of chain ends produced by irradiation is therefore $2 m r D$. If we assume each segment undergoes one scission at most at the center of the segment, the crystal involves $2 m r D$ segments with free end and $[m / n-m r D]$ normal segments. Therefore, the number average of segment length after irradiation with a dose $D, n(D)$, is given by

$$
n(D)=\frac{n\left[\frac{m}{n}-m r D\right]+\frac{n}{2} 2 m r D}{\left[\frac{m}{n}-m r D\right]+2 m r D}=\frac{n}{1+n r D}
$$

Since $T_{\mathrm{t}}$ after irradiation is proportional to $n(D)$,

$$
T_{\mathrm{t}}(D)=\frac{T_{\mathrm{t}}(0)}{1+n r D}
$$

where $T_{t}(0)$ is the transition temperature at $D=0$.

In deriving eq 6 , we assume the crystal does not consist of two kinds of segments, $n$ and $n / 2$, but consists of segments of the average length $n(D)$. This is reasonable because the value of $n$ in eq 4 is only a statistical average and a chain in the crystal involves various modes of rotational vibration with different wavelengths.

\section{Effect of the Occlusion of Degradation Products on the Transition Temperature}

When the degradation products occupy interstitial sites in the crystal lattice in PTFE, they play a role of pinning points to the rotational motion of the segments and increase the interchain force constant $g$ in eq 4. The interchain force constant at the pinned site is termed $g^{\prime}$ and that at the normal site $g$. If we assume the average interchain force constant $\bar{g}$ is given by the average

$$
\frac{1}{\bar{g}}=\frac{1}{g}\left(1-X_{\mathrm{d}}\right)+\frac{1}{g^{\prime}} X_{\mathrm{d}}
$$

then, the average of segment length in the crystal including pinning points may be written as

$$
n\left(X_{\mathrm{d}}\right)=\sqrt{\frac{f}{g}\left(1-X_{\mathrm{d}}\right)+\frac{f}{g^{\prime}} X_{\mathrm{d}}}
$$

where $X_{\mathrm{d}}$ is the fraction of pinned sites. If $g^{\prime}$ is assumed to be much larger than $g$,

$$
n\left(X_{\mathrm{d}}\right) \simeq \sqrt{\frac{f}{g}\left(1-X_{\mathrm{d}}\right)}=n \sqrt{1-X_{\mathrm{d}}}
$$

Then, from eq 3, $T_{\mathrm{t}}$ as a function of $X_{\mathrm{d}}$ becomes

$$
T_{\mathrm{t}}\left(X_{\mathrm{d}}\right)=T_{\mathrm{t}}(0) \sqrt{1-X_{\mathrm{d}}}
$$

where $T_{\mathrm{t}}(0)$ is the transition temperature for $X_{\mathrm{d}}=0$.

$X_{\mathrm{d}}$ may be estimated from the change in molecular-weight distribution as degradation progresses. The degradation theory ${ }^{20}$ predicts that, after degradation by random main-chain scission, the distribution of chain length in a polymeric system tends to be Poisson-like, i.e.,

$$
Q(p)=\frac{N}{p_{0}{ }^{2}} \exp \left(-\frac{p}{p_{0}}\right)
$$

where $Q(p)$ is the number of chains of degree of polymerization $p, N$ is the normalization factor and $p_{0}$ is the number-average degree of polymerization. If, for simplicity, the above functional form is employed, as the initial molecularweight distribution, the number of molecules which have the degree of polymerization $p$ after irradiation with a dose $D$ is given by ${ }^{20}$

$Q(p, D)=N\left(\frac{1}{p_{0}}+r D\right)^{2} \exp \left[-\left(\frac{1}{p_{0}}+r D\right) p\right]$

Then the weight fraction of the molecules which have degrees of polymerization lower than $p_{1}$ is given by

$$
\begin{aligned}
W & =\frac{\int_{0}^{p_{1}} Q(p, D) p \mathrm{~d} p}{\int_{0}^{\infty} Q(p, D) p \mathrm{~d} p} \\
& =1-\left[\left(\frac{1}{p_{0}}+r D\right) p_{1}+1\right] \exp \left[-\left(\frac{1}{p_{0}}+r D\right) p_{1}\right]
\end{aligned}
$$




\section{TUtiYA}

The degradation products with $p$ 's lower than $p_{1}$ are assumed to occupy the interstitial sites in the crystal. Since $p_{1}$ is rather small, the exponential term on the right-hand side of eq 13 can be approximated by the first term in a series expansion. Equation 13 approximates to the form

$$
W \simeq\left(p_{1} r\right)^{2} D^{2}
$$

In this approximation, it is assumed that $1 / p_{0} \ll$ $r D \ll 1 / p_{1} . \quad X_{\mathrm{d}}$ in eq 10 is proportional to $W$ in eq 14 .

$$
X_{\mathrm{d}}=C W
$$

where $C$ is a constant. If a $\mathrm{CF}_{2}$ group of the degradation products works as a pinning point of two $\mathrm{CF}_{2}$ 's in neighbor chains, $C$ is equal to 2 .

When the effects of chain scission and degradation products take place simultaneously, the transition temperature as a function of dose is given through eq $6,10,14$ and 15 by

$$
T_{\mathrm{t}}(D)=\frac{T_{\mathrm{t}}(0)}{1+n r D} \sqrt{1-C\left(p_{1} r\right)^{2} D^{2}}
$$

\section{DISCUSSION}

\section{Room-Temperature Irradiation with Subsequent Annealing}

The values of $n r$ and $T_{\mathrm{t}}(0)$ in eq 6 can be calculated from the experimental data by fitting a straight line to plot of $1 / T_{\mathrm{t}}(D)$ vs. $D$. Figure 2 shows $10^{3} / T_{\mathrm{t}}(D)$ as a function of total dose for PTFE samples irradiated and subsequently annealed in the vicinity of their melting point in air. The values of $n r$ and $T_{\mathrm{t}}(0)$ are estimated to

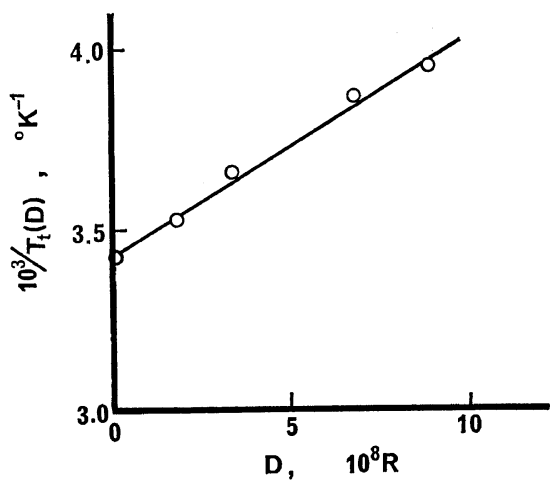

Figure 2. $10^{3} / T_{t}(D) v s$. total dose for PTFE samples irradiated in air and subsequently annealed. be $1.8 \times 10^{-10} \mathrm{R}^{-1}$ and $292^{\circ} \mathrm{K}$ from the slope and the intercept of a straight line in Figure 2. Then, the decrease in $T_{\mathrm{t}}$ of the annealed samples with increasing dose is expressed by

$$
T_{\mathrm{t}}(D)=\frac{292}{1+1.8 \times 10^{-10} D}
$$

where $T_{\mathrm{t}}(D)$ is the transition temperature in ${ }^{\circ} \mathrm{K}$ and $D$ is the radiation dose in $R$. The line $A$ in Figure 1 shows the calculated values of eq 17.

The $G$ value, which is defined by the number of events per $100-\mathrm{eV}$ energy deposition, is related with the probability $r$ as ${ }^{8}$

$$
G=1.9 \times 10^{-14} \frac{N_{\mathrm{A}}}{w} r
$$

where $N_{\mathrm{A}}$ is the Avogadro number and $w$ is the molecular weight of a $\mathrm{CF}_{2}$ group.

Using the value of $n r=1.8 \times 10^{-10}$ along with $n=4,{ }^{18}$ the $G$ value is calculated as 1.1 . The $G$ value of $\mathrm{C}-\mathrm{C}$ scission reported in the literatures $^{6,8,21}$ ranges from 0.3 to 10 . Recently, the $G$ value was estimated by Kusy and Turner ${ }^{10}$ to be $1.6 \pm 0.2$ from the lowering of $T_{\mathrm{t}}$ with increasing dose up to $10^{8} \mathrm{R}$. They assumed Flory's equation $^{22}$ for the lowering of transition temperature due to radiation-induced defects. These facts indicate that the present value of $G=1.1$ is a reasonable estimate.

\section{Room-Temperature Irradiation}

In the previous paper, ${ }^{9}$ the lowering of $T_{\mathrm{t}}$ of the irradiated samples (prior to annealing) was attributed to the combined effect of main-chain scission and conclusion of degradation products.

The value of $C\left(p_{1} r\right)^{2}$ in eq 16 can be calculated from the experimental data by fitting a straight line to plot of $\left[\left(T_{\mathrm{t}}(D)(1+n r D) / T_{\mathrm{t}}(0)\right]^{2}\right.$ vs. $D^{2}$.

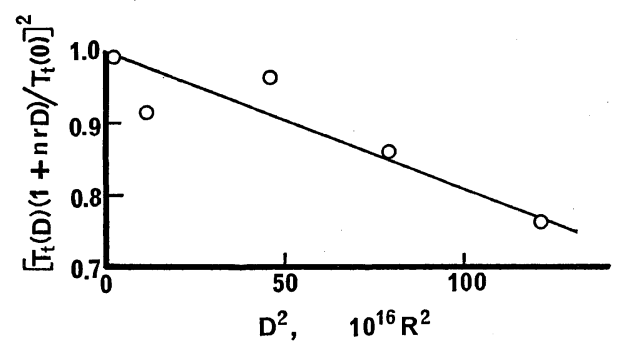

Figure 3. $\left[\left(T_{\mathrm{t}}(D)(1+n r D)\right) / T_{\mathrm{t}}(0)\right]^{2} \quad$ vs. square of total dose for PTFE samples irradiated at room temperature in air. 
Figure 3 shows $\left[T_{t}(D)(1+n r D) / T_{t}(0)\right]^{2}$ as a function of $D^{2}$ for PTFE samples irradiated in air. The value of $C\left(p_{1} r\right)^{2}$ is estimated to be $20 \times$ $10^{-20} \mathrm{R}^{-2}$ from the slope of a straight line in Figure 3. The observed lowering of the crystalline transition temperature in the irradiated samples is then approximately expressed by

$$
T_{\mathrm{t}}(D)=\frac{292}{1+1.8 \times 10^{-10} D} \sqrt{1-20 \times 10^{-20} D^{2}}
$$

The line B in Figure 1 indicates values calculated from eq 19.

If a $\mathrm{CF}_{2}$ group of the interstitial degradation products works as a pinning point for two $\mathrm{CF}_{2}$ groups, each belonging to neighboring mainchains, $C$ in eq 16 is to be taken as 2 . By use of values $C=2$ and $G=1.1, p_{1}$ is estimated to be 7. This means that the degradation products which have degrees of polymerization lower than 7 occupy the interstitial sites in the crystal. These products are evaporated from the crystal with annealing. In accordance with this conclusion, Danno, et al. ${ }^{23}$ suggested that the degradation products smaller than $C_{13}$ perfluorocarbons in the irradiated PTFE are evaporated by annealing.

The assumption $1 / p_{0} \ll r D \ll 1 / p_{1}$ was used in the derivation of $p_{1}$ in eq 16 . Therefore, eq 19 is applicable to the dose range of $10^{6} \mathrm{R} \ll D \ll 10^{10} \mathrm{R}$. According to Kline, et al.,$^{5,8}$ the lowering of $T_{\mathrm{t}}$ in the above dose range is experimentally expressed by

$$
T_{t}(D)=292 \exp \left[-2.5 \times 10^{-4} D\right]
$$

where $D$ is the radiation dose in Mrad. The dashed line in Figure 1 shows eq 20.

\section{Irradiation at High Temperatures}

It has been suggested in the previous paper ${ }^{13}$ that the effect of the main-chain scission on the lowering of $T_{\mathrm{t}}$ may be negligible up to $10^{8} \mathrm{R}$ and that the remarkable decrease in $T_{\mathrm{t}}$ of the PTFE samples irradiated at $340^{\circ} \mathrm{C}$ below $10^{8} \mathrm{R}$ in vacuum-sealed glass tubes may arise mainly due to the occlusion of degradation products and to the formation of side-branches $\left(-\mathrm{CF}_{3}\right)$.

In Table $\mathrm{I}$, a close correlation is found between the weight fraction of HFP ( $\left.W_{\mathrm{HFP}}\right)$ and the lowering of $T_{\mathrm{t}}$ with irradiation. This is in a good agreement with the data by $\mathrm{McCrum}^{24}$ or Boltz and $\mathrm{Eby}^{25}$ who showed the decrease in the crystalline transition temperature with increasing

\section{HFP contents.}

We assume that the decrease in $T_{\mathrm{t}}$ with increasing HFP contents can be expressed by eq 10 .

$$
T_{\mathrm{t}}\left(W_{\mathrm{HFP}}\right)=292 \sqrt{1-C^{\prime} W_{\mathrm{HFP}}}
$$

The data by $\mathrm{McCrum}^{24}\left(T_{\mathrm{t}}\left(W_{\mathrm{HFP}}\right)=240^{\circ} \mathrm{K}\right.$ at $\left.W_{\text {HFP }}=5.5 \%\right)$ give the proportional constant $C^{\prime}$ as 6. From the values of $W_{\mathrm{HFP}}$ in Table $\mathrm{I}, T_{\mathrm{t}}$ was calculated to be $287^{\circ} \mathrm{K}$ at $330^{\circ} \mathrm{C}$ irradiation with $1.1 \times 10^{7} \mathrm{R}$ and $268^{\circ} \mathrm{K}$ at $300^{\circ} \mathrm{C}$ irradiation with $6.2 \times 10^{7} R$, which exhibit considerable discrepancies from the observed values in Table $I$, $273^{\circ} \mathrm{K}$ for both cases. The calculated lowering of $T_{\mathrm{t}}$ is larger than the observed one at the irradiation above the melting point $T_{\mathrm{m}}$ but smaller at the irradiation below $T_{\mathrm{m}}$.

Table I shows that the side-branches $\left(-\mathrm{CF}_{3}\right)$ are easily formed by irradiation above $T_{\mathrm{m}}\left(327^{\circ} \mathrm{C}\right)$ where the crystalline phase of PTFE is changed into the liquid or paracrystalline phase. ${ }^{26}$ This indicates that the side-branches are easily formed under the following conditions: (a) PTFE molecules above $T_{\mathrm{m}}$ are to some degree flexible so that they can change their conformation, and (b) the viscosity is so high even above $T_{\mathrm{m}}$ that the reactive species may be trapped within PTFE samples. The discrepancy in $T_{\mathrm{t}}$ between the experimental value $\left(273^{\circ} \mathrm{K}\right)$ and the calculated one from $W_{\mathrm{HFP}}\left(268^{\circ} \mathrm{K}\right)$ in case of irradiation below $T_{\mathrm{m}}$ may possibly be due to the fact that the side-branches are formed mainly in the amorphous phase rather than in the crystalline one and are ineffective for the lowering of $T_{\mathrm{t}}$.

The formation of side-branches is necessarily accompanied with the production of degradation products. In case of the irradiation with electrons at temperatures above $T_{\mathrm{m}}$ in air, ${ }^{27}$ the depropagation processes takes place as suggested by Florin and Wall. ${ }^{21}$

$$
\begin{gathered}
-\mathrm{CF}_{2} \mathrm{CF}_{2} \mathrm{CF}_{2} \mathrm{CF}_{2} \mathrm{CF}_{2} \mathrm{CF}_{2} \cdot \rightarrow-\mathrm{CF}_{2} \mathrm{CF}_{2} \mathrm{CF}_{2} \mathrm{CF}_{2} \cdot \\
+\mathrm{C}_{2} \mathrm{~F}_{4} \rightarrow-\mathrm{CF}_{2} \mathrm{CF}_{2} \cdot+2 \mathrm{C}_{2} \mathrm{~F}_{4}
\end{gathered}
$$

In this case, the $G$ value of degradation products $\left(\mathrm{C}_{2} \mathrm{~F}_{4}\right)$ has been estimated to be $c a .30 .^{21}$ Taking this into consideration, the occlusion of degradation products may be also effective for the lowering of $T_{\mathrm{t}}$ in the irradiation above $T_{\mathrm{m}}$.

Assuming that the discrepancy in $T_{\mathrm{t}}$ between experiment $\left(273^{\circ} \mathrm{K}\right)$ and theory $\left(287^{\circ} \mathrm{K}\right)$ in the case of $330^{\circ} \mathrm{C}$ irradiation with $1.1 \times 10^{7} \mathrm{R}$ is due 


\section{Tutiya}

to the occlusion of the degradation products, the $G$ value of degradation products $\left(\mathrm{C}_{2} \mathrm{~F}_{4}\right)$ is estimated to be $c a$. 50. Such a high $G$ value is unreasonable, because the present experiment has been carried out with ${ }^{60} \mathrm{Co} \gamma$-rays in vacuum. Some factors ${ }^{13}$ other than the occlusion of degradation products may be involved.

Double bonds, ${ }^{28}$ crosslinkings and others may possibly occur due to irradiation. If these occur in the crystal lattice, their effects on $T_{\mathrm{t}}$ must be taken into account. At present, however, these effects could not be verified.

Acknowledgements. The author wishes to thank Professor Y. Wada at the University of Tokyo for his continuing guidance throughout this work and Dr. K. Yamamoto at Tokyo Metropolitan Isotope Research Center for his encouragement.

\section{REFERENCES}

1. K. Shinohara and H. Kashiwabara, "Hosyasen to Kobunshi (Radiation and Polymers)," MakiShoten, Tokyo, 1968, p 90.

2. M. Tutiya and K. Yamamoto, Oyo Buturi, 34, 424 (1965).

3. M. Tutiya, J. Phys. Soc. Japan, 25, 1518 (1968).

4. W. R. Licht and D. E. Kline, J. Polym. Sci. Part A-2, 4, 343 (1966).

5. G. A. Bernier, D. E. Kline and J. A. Sauer, $J$. Macromol. Sci. (Phys.), B1, 335 (1967).

6. A. Nishioka, K. Matsumae, M. Watanabe, M. Tajima and M. Owaki, J. Appl. Polym. Sci., 11, 114 (1959).

7. W. M. Peffely, V. R. Honnold and D. Binder, J. Polym. Sci. Part A-1, 4, 977 (1966).
8. W. R. Licht and D. E. Kline, ibid. Part A-2, 2, 4673 (1964).

9. M. Tutiya, Japan. J. Appl. Phys., 9, 1204 (1970).

10. R. P. Kusy and D. T. Turner, J. Polym. Sci. Part A-1, 10, 1745 (1972).

11. K. L. Hsu, D. E. Kline and J. N. Tomlinson, J. Appl. Polym. Sci., 9, 3567 (1965).

12. T. Ishibashi, M. Takeuchi and A. Odajima, Rep. Progr. Polym. Phys. Japan, 15, 233 (1972).

13. M. Tutiya, Japan. J. Appl. Phys., 11, 1542 (1972).

14. D. Hyndman and G. F. Origlio, J. Appl. Phys., 31, 1849 (1960).

15. T. Satokawa, Y. Kometani, A. Yamada and S. Koizumi, "Husso Zyushi (Fluoro Resins)," Nikkankogyo-Shinbunsya, Tokyo, 1969, p 190.

16. Y. Wada, "Kobunshi no Kotaibussei (Polymer Physics at Solid State)," Baihukan, Tokyo, 1971, p 220.

17. J. D. Hoffman, J. Chem. Phys., 20, 541 (1952).

18. Y. Wada, A. Itani, T. Nishi and S. Nagai, $J$. Polym. Sci. Part A-2, 7, 201 (1969).

19. A. Itani and Y. Wada, Rep. Progr. Polym. Phys. Japan, 12, 293 (1969).

20. O. Saito, J. Phys. Soc. Japan, 13, 198 (1958).

21. R. E. Florin and L. A. Wall, J. Res. Nat. Bur. Stand., 65A, 375 (1961).

22. P. J. Flory, J. Chem. Phys., 17, 223 (1949).

23. A. Danno, M. Koike, K. Doi and M. Inoue, Rep. Progr. Polym. Phys. Japan, 5, 270 (1962).

24. N. G. McCrum, Makromol. Chem., 34, 50 (1959).

25. L. H. Boltz and R. K. Eby, J. Res. Nat. Bur. Stand. A, 69, 481 (1965).

26. Y. Ohzawa and Y. Wada, Japan J. Appl. Phys., 3, 436 (1964).

27. A. Chapiro, "Radiation Chemistry of Polymeric System," Interscience, London, 1962, p 527.

28. J. W. Ryan, Modern Plastics, 152 (1953). 